\title{
Psoriasis vulgaris and digestive system disorders: Is there a linkage?
}

\author{
Aldona Pietrzak ${ }^{1}$, Iwona Jastrzębska ${ }^{2}$, Grażyna Chodorowska', \\ Ryszard Maciejewski ${ }^{3}$, Ewa Dybiec ${ }^{4}$, Maria Juszkiewicz-Borowiec ${ }^{1}$, \\ Dorota Krasowska ${ }^{1}$, Robert A. Schwartz ${ }^{5}$
}

\author{
${ }^{1}$ Department of Dermatology, Venereology, and Paediatric Dermatology, Medical University of Lublin, \\ Poland \\ ${ }^{2}$ Chair of Internal Medicine and Department of Internal Nursing, Medical University of Lublin, Poland \\ ${ }^{3}$ Ist $^{\text {st }}$ Medical Faculty with Dentistry Division, IInd Medical Faculty with English Division, Pharmaceutical \\ Faculty with Medical Analytic Division, Nursing and Health Sciences Faculty, Medical University \\ of Lublin, Poland \\ ${ }^{4}$ Department of Paediatric Radiology, Medical University of Lublin, Poland \\ 5 Professor and Head of Dermatology, Professor of Medicine, Professor of Paediatrics, Professor \\ of Pathology, Professor of Preventive Medicine and Community Health, University of Medicine \\ and Dentistry, New Jersey Medical School, Newark, United States of America
}

\begin{abstract}
Psoriasis is well-known immune-mediated skin disease often associated with co-morbidities, including dyslipidaemia and obesity. Few reports imply that the disease might be also related to pathology of mucosal surfaces, especially that of the digestive system. The authors present a case of psoriasis and concurrent digestive system abnormalities, and review the literature regarding the topic. A 40-year-old man suffered from an exacerbation of exudative psoriasis for about 6 months. Topical antipsoriatics proved ineffective and the disease gradually progressed to a severe disseminated form. Subsequent detailed examinations revealed persistent gastroduodenitis due to $H$. pylori infection, pancreatic dysfunction and fatty change of the liver, although the patient denied any gastrointestinal symptoms. As a result appropriate treatment of the diagnosed digestive system disorders was added to topical antipsoriatic therapy. Within 2 weeks of treatment clinical symptoms and laboratory signs showed a marked trend to normalisation. The presented medical history seems to suggest that there may be some kind of interplay between psoriasis and digestive system disorders.
\end{abstract}

Key words: psoriasis; digestive system; gastritis, pancreatitis; chronic liver disease

\section{Introduction}

Psoriasis is an immune-mediated disease of skin and joints often precipitated by infection. Cutaneous involvement is always the predominant feature. However, a couple of reports suggest that there might be also a connection between psoriasis and other organ disorders, including that of the digestive system [1].

A relationship between Helicobacter pylori infection and skin disease, especially chronic urticaria, have been documented. Helicobacter pylori eradication

Correspondence: I. Jastrzebska, Chair of Internal Medicine and Department of Internal Nursing, Medical University of Lublin, Jaczewskiego 8, 20-950 Lublin, Poland;

tel./ fax.: (+4881) 7425825, e-mail: ivjastrzebska@wp.pl appears to be beneficial in patients with lichen planus, prurigo, Sjögren's syndrome, Henoch-Schönlein purpu$\mathrm{ra}$, and rosacea [2,3]. Moreover, there are hypotheses implicating the pathogen in triggering psoriasis [3]. There are also data concerning concurrent presentation of psoriasis and gastritis, duodenitis, celiac disease or inflammatory bowel disease [4-6]. A few authors have related psoriasis and disturbances of the incretory and excretory pancreatic functions and even acute pancreatitis [7-9]. Single reports on liver dysfunction or chronic liver disease in psoriatic individuals have been published, including hepatic cirrhosis or psoriasis being a cutaneous sign of hepatocellular carcinoma [10-14].

Disposition to psoriasis is primarily genetic, but some dysmetabolic changes, such as obesity, dyslipi- 

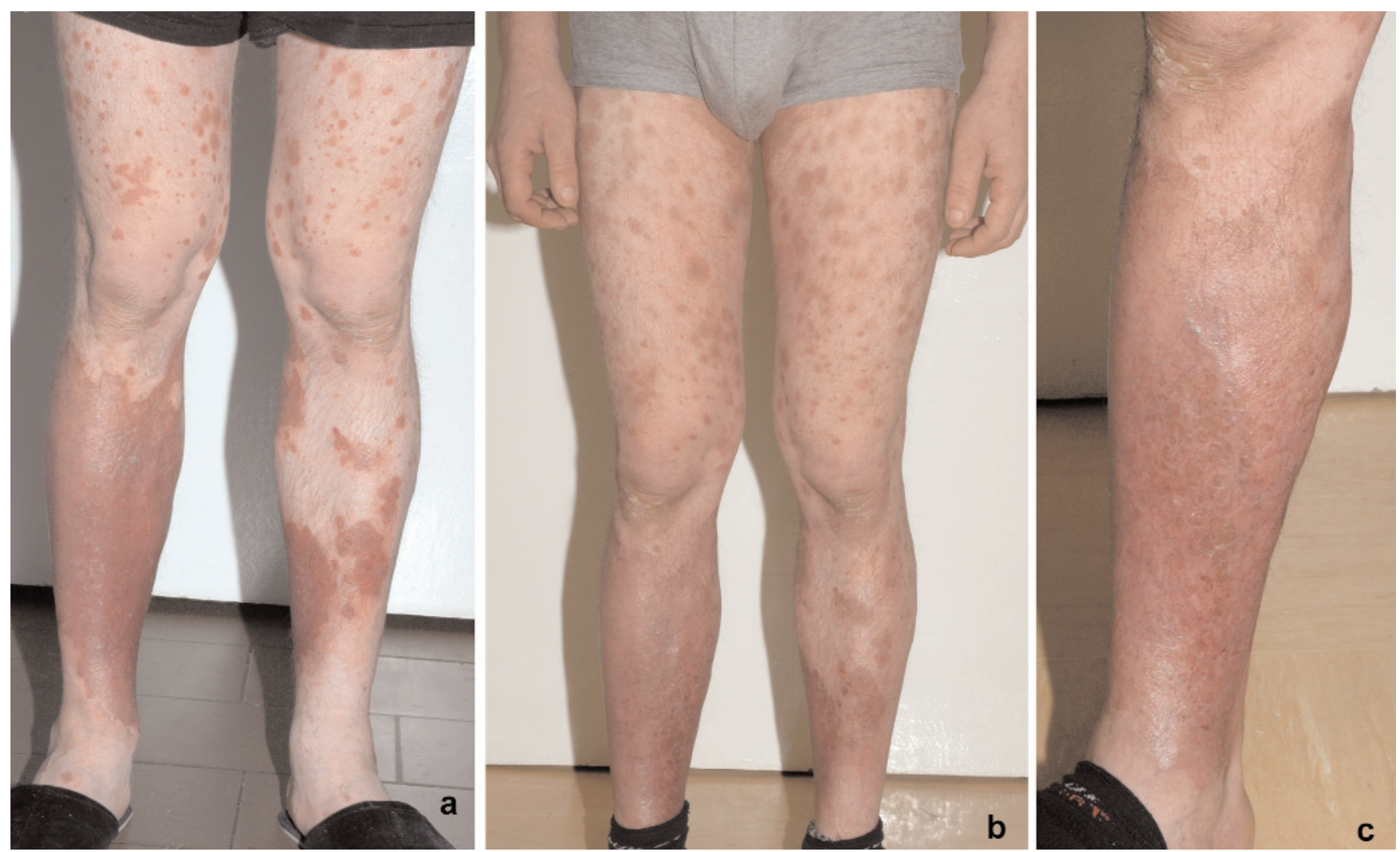

Fig. 1. Patient's lower extremities before (a) and after 2 weeks of treatment $(\mathbf{b}, \mathbf{c})$. Detailed description in text.

daemia, hyperuricaemia, impaired glucose tolerance or insulin resistance, may increase the risk for psoriasis as well as for some of the digestive system disorders, especially liver diseases [10-11]. On the other hand, pro-inflammatory factors upregulated in psoriasis, especially interleukin (IL) 18 , are likely to increase risk of the metabolic syndrome [15]. Previous reports have endorsed an association between psoriasis and the metabolic syndrome or lipid abnormalities $[6,16,17]$. Furthermore, psoriasis is believed to be an independent risk factor for subclinical atherosclerosis [6]. At present the lipid profile, lipoprotein, apolipoprotein, brain (B-type) natriuretic peptide (BNP) and its metabolite, N-terminal pro-B-type natriuretic peptide (NT-proBNP), levels are considered to be good predictors of cardiovascular disease and may be useful markers of cardiovascular risk in psoriatics $[6,18]$.

The association between psoriasis and digestive system disorders remains unclear. The current report present a case of psoriasis associated with multiple digestive system disorders and review the literature regarding the topic.

\section{Case presentation}

A 40-year-old man presented with signs and symptoms consistent with severe and disseminated exudative psoriasis. The patient had been diagnosed as having psori- asis at 8 years of age, and for 32 years he had been presenting with only few small skin plaques with no need for systemic treatment or hospitalisation. Approximately 6 months preceding admission he suddenly developed a severe exacerbation of the disease. He was treated with various topical corticosteroid preparations, such as fluticasone, clobetasol or betamethasone with salicylic acid ointments (subsequently), and short-term oral doxycycline $(2 \times 100 \mathrm{mg} / \mathrm{d} ; 10$ days $)$ and niacin $(3 \times 200 \mathrm{mg} / \mathrm{d})$. However, the treatment proved ineffective and his skin condition gradually worsened, especially following a flu-like illness 2-3 weeks prior to the admission. On admission the patient suffered from intense pruritus within the involved skin and malaise. He made no other complaints; he denied any prior or current gastrointestinal symptoms. His medical history included hypertension for more than 3 years, and his daily medication consisted of perindopril $(1 \times 5 \mathrm{mg} / \mathrm{d})$. However, due to normal blood pressure values (mean $135 / 70 \mathrm{mmHg}$ ) for more than 2 months, the antihypertensive drug had been withdrawn. The patient did not have any history of blood transfusion or habitual alcohol consumption, and had not ingested alcohol or any drugs known to induce pancreatitis or liver damage prior to the admission. There was substantial tobacco consumption habit, up to 30 cigarettes per day. A family history of psoriasis was apparent; the patient's mother suffered from the disease since childhood. Of 
note, the patient had neither familial nor personal history of gastrointestinal disorders.

On the admission psoriatic lesions covered approximately $40 \%$ of the cutaneous surface, including the scalp, trunk, buttocks, and upper and lower extremities, but not the face, neck, palms and soles. There were a large number of patches, ranging in size from 1 to $10 \mathrm{~cm}$ in diameter and having a tendency to confluence. The most prominent lesions were present on the right shin, involving the entire anterior and lateral aspect of the limb. They were very distinctive, full red colour with a violaceous tint and covered with fine scales (Fig. 1a). The psoriatic plaques on medial aspect of the left shin, as well as the lesions on medial surfaces of both arms and forearms displayed the trend to coalesce into large patches, up to man's hand size. Small water drop-shaped or coin-shaped plaques up to large patches covered with medium scales were observed on the other involved areas. The thighs were the exception with the lesions inflamed, thick and multilayered associated with overlapping scales resembling rupees. The PASI score was estimated at 28.7. Physical examination demonstrated overweight, body mass index was calculated at $29.76 \mathrm{~kg} / \mathrm{m}^{2}$. There was no evidence of any bacterial or viral infection.

Laboratory data (Table 1) revealed a remarkable elevation of serum lipase and amylase activity and abnormal liver function tests. The levels of total cholesterol and LDL cholesterol were slightly increased, whereas the serum concentrations of triglyceride and HDL cholesterol were within normal range. More detailed analysis disclosed abnormal ApoB/ApoA-I ratio. In addition, there were significant increases in serum level of CRP (C reactive protein) and NT-proBNP. All virologic and serologic markers for hepatitis $\mathrm{B}$ and $\mathrm{C}$ virus were negative. An abdominal ultrasound revealed slight hepatomegaly and inhomogeneous fatty change of the liver (Fig. 2). No stones or sludge of the gallbladder and of the common bile duct were detected. The pancreas was homogeneous and measured $25 \mathrm{~mm}$ at the head, $26 \mathrm{~mm}$ at the body, and $29 \mathrm{~mm}$ at the tail. An upper gastrointestinal tract endoscopy displayed no macroscopic pathology of the oesophagus, stomach or duodenum. H. pylori infection was diagnosed with a biopsy check during endoscopy with a rapid urease test. Histological examination of gastric and duodenal mucosa biopsies disclosed significant inflammatory changes (Fig. 3). Despite remarkable disturbances of serum lipid profile, there were no significant abnormalities on cardiological examination. The transthoracic echocardiography revealed only negligible dilatation of the left atrium and ventricle and slight left ventricle hypertrophy, but there were no changes of the left ventricle ejection fraction.

Initially topical treatment for psoriasis consisted of dexpanthenol aerosol. Subsequently it was continued

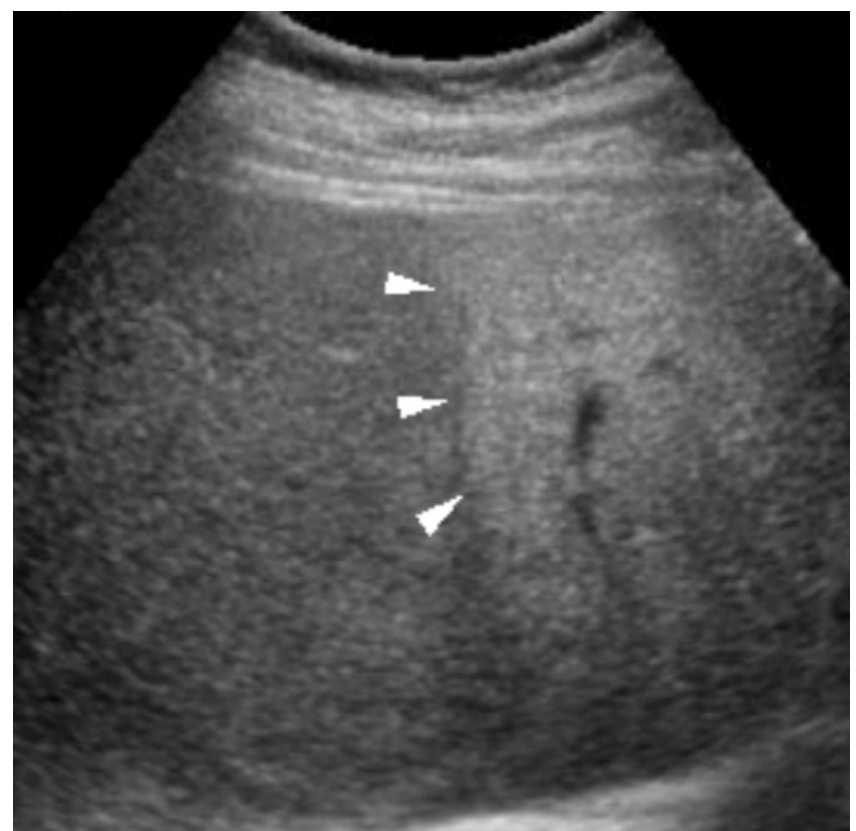

Fig. 2. Liver ultrasound scan. Inhomogeneous increase of the liver echogenicity within the segment 4 of the right lobe (arrowheads), the segment 7 of the right lobe and the segments 2 and 3 of the left lobe. Hepatomegaly; the liver size in the anterior axial line was $146 \mathrm{~mm}$; in the midclavicular line, $119 \mathrm{~mm}$; and in the sternal line, $84 \mathrm{~mm}$.

with corticosteroid preparations, including hydrocortisone ointment, betamethasone with salicylic acid ointment and then fluticasone cream, combined with $0.125-0.25 \%$ cignolin, cholesterol, urea with salicylic acid, salicylic acid sulfur soap, or sulfur salicylic acid ointments. In addition, oral antihistamine therapy with clemastine $(2 \times 1 \mathrm{mg} / \mathrm{d})$ for 10 days and subsequently with fexofenadine $(1 \times 180 \mathrm{mg} / \mathrm{d})$ was introduced. Therapeutic measures for gastrointestinal disorders included appropriate diet and pancrelipase $(10,000 \mathrm{IU}$ per meal or snack; $3 \times / \mathrm{d}$ ), as well as hepatoprotectants, such as thiazolidine-4-carboxylic acid $(3 \times 200 \mathrm{mg} / \mathrm{d})$ and essential phospholipids, especially 3 -sn-phosphatidylcholine $(2 \times 300 \mathrm{mg} / \mathrm{d})$. The patient received vitamin $\mathrm{E}(3 \times 100 \mathrm{mg} / \mathrm{d})$, and ascorbic acid combined with rutoside $(3 \times 200+50 \mathrm{mg} / \mathrm{d})$ as well. Moreover, with the $H$. pylori infection diagnosis the standard treatment for the pathogen eradication was initiated, consisting of amoxycillin $(2 \times 1 \mathrm{~g} / \mathrm{d} ; 10$ days $)$ and clarithromycin $(2 \times 500 \mathrm{mg} / \mathrm{d} ; 14$ days $)$ combined with omeprazole $(2 \times 20 \mathrm{mg} / \mathrm{d} ; 14$ days $)$. Within 2 weeks of the treatment a considerable clinical improvement was achieved. The itching subsided. The skin lesions started to remit gradually. At the time of discharge psoriatic scales persisted only on the skin of knee-joints and right shin, at the other sites previously covered with lesions there were distinct post-inflammatory hyperpigmentations (Fig. 1b, c). Simultaneously trend to normalisation of the pancreas and liver function parameters was observed (Table 1). 
Table 1. Clinical characteristic of the patient (normal reference ranges are given in parentheses; $*$ for individuals younger than 75 years).

\begin{tabular}{|c|c|c|c|c|}
\hline & & On admission & During treatment & At discharge \\
\hline PASI score & & 28.70 & & 19.20 \\
\hline \multicolumn{5}{|l|}{ Peripheral blood } \\
\hline Red blood cell count $\left(\mathrm{M} / \mathrm{mm}^{3}\right)$ & $(4.00-5.70)$ & 3.94 & & \\
\hline Haematocrit level (\%) & $(40-54)$ & 40.60 & & \\
\hline Haemoglobin $(\mathrm{g} / \mathrm{dL})$ & $(13-18)$ & 13.30 & & \\
\hline $\mathrm{MCV}(\mathrm{fL})$ & $(80-100)$ & 103 & & \\
\hline White blood cell count $\left(\mathrm{K} / \mathrm{mm}^{3}\right)$ & $(4-10)$ & 7.70 & & \\
\hline Thrombocyte count $\left(\mathrm{K} / \mathrm{mm}^{3}\right)$ & $(120-400)$ & 341 & & \\
\hline \multicolumn{5}{|l|}{ Serum chemistry } \\
\hline $\mathrm{CRP}(\mathrm{mg} / \mathrm{dL})$ & $(0-10)$ & 23.70 & & 21.50 \\
\hline Total bilirubin (mg/dL) & $(0.20-1.10)$ & 0.72 & & \\
\hline Glucose (mg/dL) & $(65-100)$ & 92 & & \\
\hline Uric acid (mg/dL) & $(3-7)$ & 4.28 & & \\
\hline Total cholesterol (mg/dL) & $(120-200)$ & 238 & & 194 \\
\hline HDL cholesterol (mg/dL) & $(35-60)$ & 45 & & 34 \\
\hline LDL cholesterol (mg/dL) & $(70-135)$ & 173 & & 127 \\
\hline Triglyceride (mg/dL) & $(40-160)$ & 100 & & 164 \\
\hline ApoA-I (g/L) & $(1.10-2.10)$ & 1.15 & & 0.81 \\
\hline ApoB (g/L) & $(0.50-1.40)$ & 1.31 & & 1.11 \\
\hline ApoB/ApoA-I & $(\sim 0.50)$ & 1.13 & & 1.37 \\
\hline NT-proBNP (pg/mL) & $(110-125)^{*}$ & 660 & & 752 \\
\hline Homocystein $(\mu \mathrm{mol} / \mathrm{L})$ & $(5-12)$ & & & 8.43 \\
\hline Pancreatic Lipase - EC 3.1.1.3 (IU/L) & $(7-60)$ & 319 & 75 & 66 \\
\hline$\alpha$-Amylase (U/L) & $(20-80)$ & 135 & & 67 \\
\hline ALT (IU/L) & $(10-40)$ & 62 & & 27 \\
\hline AST (IU/L) & $(10-40)$ & 151 & & 53 \\
\hline$\gamma$-GTP (IU/L) & $(11-50)$ & 559 & 390 & 144 \\
\hline Sodium $(\mathrm{mmol} / \mathrm{L})$ & $(135-148)$ & 143 & & 138 \\
\hline Potassium $(\mathrm{mmol} / \mathrm{L})$ & $(3.40-5.50)$ & 4.03 & & 3.80 \\
\hline Calcium (mmol/L) & $(2.10-2.60)$ & 2.19 & & \\
\hline Chlorine $(\mathrm{mmol} / \mathrm{L})$ & $(94-105)$ & 110 & & 103 \\
\hline \multicolumn{5}{|l|}{ Virus markers } \\
\hline HBs-Ag & & & absent & \\
\hline $\mathrm{HCV}-\mathrm{Ab}$ & & & absent & \\
\hline Urinalysis & & normal & normal & normal \\
\hline
\end{tabular}

ALT - alanine transferase; ApoA-I - apolipoprotein A-I; ApoB - apolipoprotein B; AST - aspartate transferase; CRP - C reactive protein; $\gamma$-GTP - $\gamma$-glutamyl transpeptidase; HBs-Ag - hepatitis B surface antigen; HCV-Ab - hepatitis C virus antibody; HDL - high density lipoprotein; IU/L - international units/liter; LDL - low density lipoprotein; MCV - mean corpuscular volume (i.e. average red blood cell size); NT-proBNP - N-terminal pro B-type natriuretic peptide; PASI - Psoriasis Area and Severity Index.

\section{Discussion}

Little is known about the potential relationship between psoriasis and gastrointestinal, pancreas and liver dysfunction. However, the digestive system dis- orders are speculated to be involved in the psoriasis pathology. The presented case, consistently with a few published reports, appears to suggest that $H$. pylori infection may be related to psoriasis. Nevertheless, the 

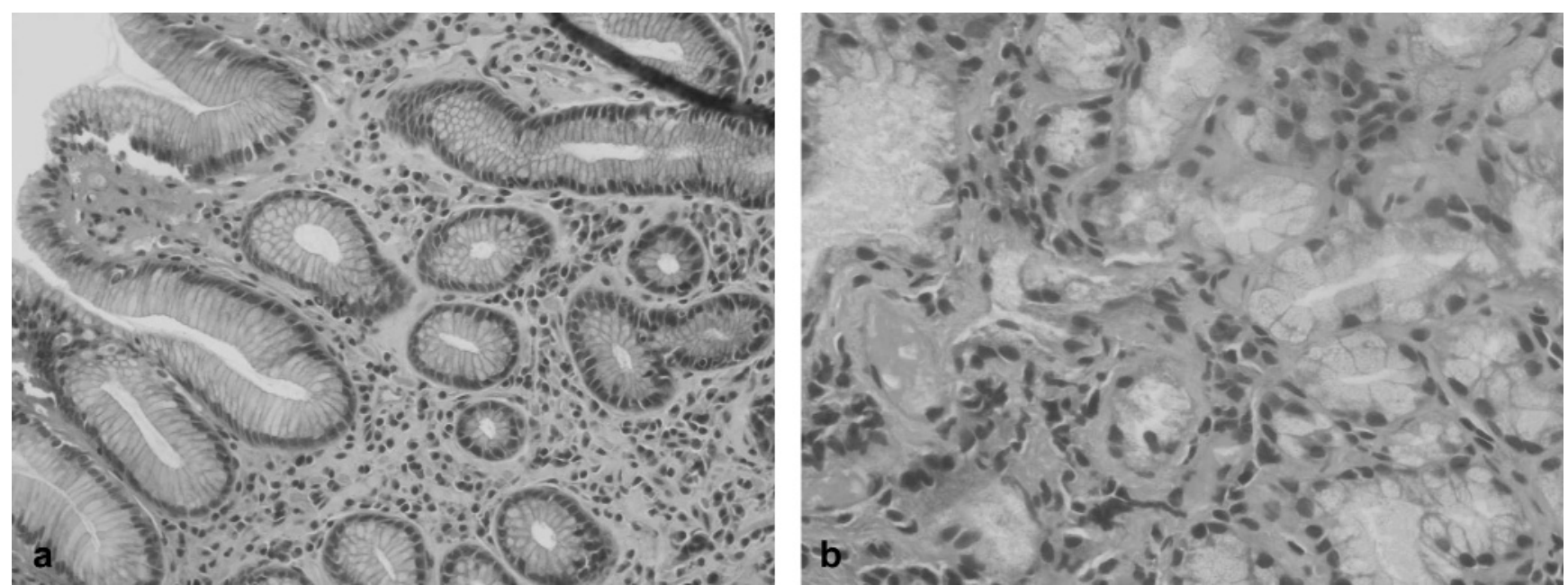

Fig. 3. Histological examination of gastric (a) and duodenal (b) biopsies (H+E). (a) Gastric mucosa with hyperaemia, oedema and moderate infiltration with plasma cells and lymphocytes as well as single eosinophils and neutrophils (original magnification, $\times 20$ ). (b) Duodenal mucosa with considerable hyperaemia and oedema, most prominent within stroma of the duodenal papilla. Moreover, there is moderately increased cellularity of the mucosa with lymphocytes and plasma cells, and single neutrophils and eosinophils (original magnification, $\times 40$ ). The histopathological examination is consistent with persistent gastritis and duodenitis, respectively.

existing literature data are conflicting. Some authors described marked improvement and/or remission of the refractory disease following proven bacteria eradication $[19,20]$, whereas others consider the approach to be of no benefit to psoriatic individuals [21]. For example, Dauden et al. [21] observed no improvement of psoriatic lesions, despite successful $H$. pylori eradication. Potential cutaneous pathology of $H$. pylori remains unknown, but it may be associated with a superantigen effect of the bacteria or their products, increased mucosal permeability of the gastrointestinal tract, various autoimmune mechanisms, or impairment of vascular integrity [2]. Recently, a hypothesis of superantigen-mediated pathogenicity has became a focus for research on psoriasis [22]. This new concept of psoriasis pathophysiology, defining it as a Th-1-mediated disorder, implicates superantigens in triggering the disease. As they bind MHC class-II molecules, not only constitutively expressed HLA-DR molecules on professional antigen-presenting cells, including macrophages and dendritic cells, but also cytokine-induced HLA-DR molecules on nonprofessional antigen-presenting cells, such as keratinocytes, without any prior processing, superantigens easily prime large number of resting T cells. Superantigeninduced $\mathrm{T}$ cells are likely to produce large amounts of inflammatory cytokines, including TNF- $\alpha$ and $-\beta$, IL-2 or INF- $\gamma[2,22]$. It is enticing to speculate that some of unique virulence factors developed by $H$. pylori to survive and proliferate in the stomach may act as superantigens (i.e. immunostimulatory molecules acting as $\mathrm{V} \beta$-restricted extremely potent polyclonal $\mathrm{T}$ cell mitogens). These may include the urease, flagellar proteins, an H-gated urea transporter, several adhesins, and espe- cially multifunctional vacuolating cytotoxin A (VacA) and the cytotoxin-associated gene pathogenicity island (cag-PAI). Of note, expression of the cag-PAI is responsible for the building of a secretion apparatus delivering some bacterial proteins, such as an immunodominant antigen cag A, directly into the cytosol of host cells $[2,23]$. However, preliminary study of Dauden et al. [23] indicated that cag A status was not associated with type, severity and duration of psoriasis. Another factor probably linking $H$. pylori infection and the psoriasis pathophysiology is increased mucosal permeability of the stomach and intestine. Abnormalities of gastric or intestinal epithelium structure and function might result in a greater exposure to alimentary antigens or toxins, including microbial factors. These may leak into circulation, evoke specific immune response and eventually may be deposited along the skin basement membrane zone, which is believed to be the first step of their percutaneous elimination. If microbial antigens share structural homologies with skin components (molecular mimicry), the additional result may be the development of antigenantibody complexes, cross-reactive antibodies or specific $\mathrm{T}$ cells cross-reacting against epidermal autoantigens $[24,25]$. For example, molecular mimicry between $H$. pylori antigens and keratin 17 may result in activation of autoreactive $\mathrm{T}$ cells and augment keratinocyte hyperproliferation [22]. Moreover, some H. pylori products or structural proteins may crossreact with such extragastrointestinal epitopes as Lewis antigens [26,27], sialylated glycoconjugates [28] or sialic residues of laminin [29]. Humbert et al. [30] findings seem to support the hypothesis. Increased mucosal permeability of the stomach and intestine has 
been demonstrated in $H$. pylori-infected subjects as well [31]. Vascular impairment is considered to be another important component of the most skin diseases linked to $H$. pylori infection. Animal studies demonstrated that the bacteria disrupted interstitial and intravascular cell to cell interactions, leading to marked microvascular dysfunction [32]. Furthermore, it is probably associated with increased levels of fibrinogen [33], and platelet activation and aggregation [34], other processes implicated in the microvascular dysfunction and inflammatory-cell recruitment.

Presented medical history may also signal the relationship between psoriasis and pancreas disorders. Intriguingly, no clinical signs or symptoms of pancreatitis were reported; especially the patient denied abdominal pain, which is a dominant feature of the disease. Literature data indicate that asymptomatic abnormalities of pancreatic enzymes are not infrequent in patients with inflammatory bowel diseases, or positive for chronic hepatitis B or C virus, or HIV infection. Most of these patients do not develop clinical pancreatitis and usually do not require specific therapy $[35,36]$. Only few reports regarding the pancreas dysfunction in psoriasis have been published $[7,8]$. Pietrzak et al. [7] demonstrated higher serum levels of pancreatic lipase in male psoriatics compared to healthy controls. Khardikova et al. [8] observed impaired exocrine and endocrine pancreas function in psoriatic individuals, the latter finding may account for higher prevalence of diabetes mellitus in psoriasis [37]. Recently recurrent acute pancreatitis over the course of psoriatic arthritis has been described [9]. In the case presented here interpretation may be biased by the patient's hypertension history and perindopril administration. One should remember that some systemic as well as topical medications were sporadically linked to acute pancreatitis [38-41] including angiotensin-converting enzyme inhibitors (i.e. perindopril) $[42,43]$. However, the patient had not presented with any signs and symptoms within prior three-year antihypertensive treatment, and due to normal blood pressure values, he had not been taking the compound for more than 2 months preceding the diagnosis of pancreatitis. Other pancreatitis causes, including mechanical obstacle or current heavy drinking, were excluded with the use of various gold-standard techniques. Possible relationship between psoriasis and pancreatitis remains an enigma. A noteworthy hypothesis could be that of intestinal barrier dysfunction, all the more since some authors reported increased intestinal permeability in patients with acute pancreatitis $[44,45]$. There is, however, another possibility. Some clinical and experimental evidence suggests that the immune system and pro-inflammatory cytokines play a key role in the pathogenesis of acute pancreatitis, with IL-18 being one of the pivotal mediators of inflammation [46]. One should appreciate that IL-18 is believed to be involved in psoriasis development and is up-regulated in psoriatic individuals $[47,48]$.

At the end of the list of digestive system pathology observed in the presented case, but not least, are liver abnormalities. The literature analysis revealed a couple of reports of recurrent cholestatic jaundice related to generalised pustular psoriasis. The jaundice episodes were of unclear pathogenesis, but some neutrophilic cholangitis was documented on liver biopsy. It raised the question whether the organ was the subject to the same disorder as the skin, inasmuch accumulation of neutrophils in the epidermis is one of the vital histological features of pustular psoriasis $[12,13]$. Apart from biliary involvement in the course of pustular psoriasis, nonalcoholic steatohepatitis was linked to psoriasis vulgaris $[10,11]$. The fatty liver change related to psoriasis vulgaris were noted in the "POLI.ST.E.N.A." study, being a prospective survey on the nonalcoholic fatty liver disease (NAFLD) [10]. The conditions coexisted in overweight or obese young adults with a central-type body fat distribution. Of note, not only is obesity believed to be associated with NAFLD, but also it has been reported to be a separate risk factor for more severe psoriasis $[6,49,50]$. Given the current trends in systemic therapy of psoriasis, especially the usage of drugs of well documented hepatotoxicity, e.g. methotrexate, the association of the disease and even modest liver damage might be of great clinical importance [51]. The pathogenetic link between psoriasis, obesity and NAFLD remains unclear. However, evolving research suggests that the chronic inflammatory nature of psoriasis itself may be an important factor for the mentioned relationships. Psoriasis is characterised by a Th1 cytokine preponderance, and these factors have pleiotropic effects on a large variety of processes, including angiogenesis, insulin signalling, adipogenesis, lipid metabolism, immune cell trafficking, or epidermal proliferation. Therefore, pro-inflammatory cytokines (e.g. TNF, IL-6, IL-18) are not only implicated in the psoriasis pathogenesis, but might cause inflammation in other organs as well [15]. For example, IL-18 was significantly upregulated in subjects with NAFLD and according to Vecchiet et al. [52] the factor might be even an important marker of liver diseases. In turn enhanced expression of TNF and IL-6 has been detected in the adipose tissue, which may implicate it in the local and systemic inflammatory responses [53].

The association between psoriasis and the metabolic syndrome gives rise to particular concern, due to a possible increase of cardiovascular risk. Indeed epidemiological studies revealed high prevalence of cardiovascular co-morbidity among psoriatic patients $[6,54]$. Refractory dyslipidaemia and other lipid profile abnormalities, especially abnormal ApoB/ApoA-I 
ratio and NT-proBNP level (heart failure marker), observed in the presented case seem to confirm described relationships and may signal the very onset of atherosclerotic vascular disease. The root cause of NT-proBNP level increase in the current case is unclear, but it may indicate the presence of cardiac dysfunction, in spite of insignificant cardiac examination. Of course, one should not overlook potential confounding factors that might significantly contribute to NT-proBNP elevation, including smoking, hypercholestrolaemia or drugs (e.g. angiotensin-converting enzyme inhibitors). Importantly, literature data demonstrated unchanged hepatic degradation of NT-proBNP in cirrhosis and no correlation was found between the marker levels and the hyperdynamic circulatory changes in cirrhotic subjects [55]. On the other hand, the levels of BNP and NT-proBNP tend to be lower in obese people, and hepatic steatosis is rather associated with low levels of NT-proBNP $[56,57]$.

The findings of this case seem to suggest some kind of interplay between psoriasis and digestive system disorders. Further studies are needed to establish if the pathogenic links exists and elucidate a possible chain of cause and effect as well as time relationships between specific disorders.

Presented in part as a poster at the 17th Congress of the European Academy of Dermatology and Venereology. Paris, 17-21 September 2008 .

Acknowledgements: The authors would like to thank Andrzej Prystupa, Chair and Department of Internal Diseases, Medical University of Lublin, Poland, for performing and interpreting gastroscopy. The authors also wish to express their gratitude to Franciszek Szubstarski, Military Hospital in Lublin, Poland, for histological examination of gastric and duodenal mucosa biopsies and taking photographs of the slides.

Disclosures: There is no conflict of interest to disclose. The research reported in the manuscript has been funded through the Medical University of Lublin, Poland, grant no. PW 170/08 and DS 167/09.

\section{References}

[1] Zachariae H. Pathologic findings in internal organs in psoriasis. Int J Dermatol. 1994;33:323-326.

[2] Wedi B, Kapp A. Helicobacter pylori infection in skin diseases: a critical appraisal. Am J Clin Dermatol. 2002;3:273-282.

[3] Qayoom S, Ahmad QM. Psoriasis and Helicobacter pylori. Indian J Dermatol Venereol Leprol. 2003;69:133-134.

[ 4] Damasiewicz-Bodzek A, Wielkoszyński T. Serologic markers of celiac disease in psoriatic patients. J Eur Acad Dermatol Venereol. 2008;22:1055-1061.

[ 5] Scarpa R, Manguso F, D'Arienzo A et al. Microscopic inflammatory changes in colon of patients with both active psoriasis and psoriatic arthritis without bowel symptoms. $J$ Rheumatol 2000;27:1241-1246.

[ 6] Kimball AB, Gladman D, Gelfand JM et al. National Psoriasis Foundation. National Psoriasis Foundation clinical consensus on psoriasis comorbidities and recommendations for screening. J Am Acad Dermatol. 2008;58:1031-1042.
[ 7] Pietrzak A, Lecewicz-Toruń B. Activity of serum lipase [EC 3.1.1.3] and the diversity of serum lipid profile in psoriasis. Med Sci Monit. 2002;8:CR9-CR13.

[ 8] Khardikova SA, Savkina KA, Kaliuzhina MI. Disturbances of the incretory and excretory pancreatic functions in patients suffering from psoriasis in combination with chronic opisthorchosis. Klin Med (Mosk). 2006;84:54-56.

[ 9] Amouzougan A, Chopin F, Patouillard B, Pallot-Prades B, Le Gars L, Thomas T. Recurrent acute pancreatitis in psoriatic arthritis. Joint Bone Spine. 2007;74:513-515.

[10] Lonardo A, Loria P, Carulli N. Concurrent non-alcoholic steatohepatitis and psoriasis. Report of three cases from the POLI.ST.E.N.A. study. Dig Liver Dis. 2001;33:86-87.

[11] Matsumoto T, Suzuki N, Watanabe H et al. Nonalcoholic steatohepatitis associated with psoriasis vulgaris. $J$ Gastroenterol. 2004;39:1102-1105.

[12] Viguier M, Allez M, Zagdanski AM et al. High frequency of cholestasis in generalized pustular psoriasis: Evidence for neutrophilic involvement of the biliary tract. Hepatology. 2004;40:452-458.

[13] Allez M, Roux ME, Bertheau P et al. Recurrent cholestatic jaundice associated with generalized pustular psoriasis: evidence for a neutrophilic cholangitis. $J$ Hepatol. 2000;33:160-162.

[14] Tsunemi Y, Ihn H, Idezuki T, Okochi H, Tamaki K. Psoriasis guttata in association with hepatocellular carcinoma. Acta Derm Venereol. 2003;83:70-71.

[15] Azfar RS, Gelfand JM. Psoriasis and metabolic disease: epidemiology and pathophysiology. Curr Opin Rheumatol. 2008;20:416-422.

[16] Cohen AD, Gilutz H, Henkin Y et al. Psoriasis and the metabolic syndrome. Acta Derm Venereol. 2007;87:506-509.

[17] Dreiher J, Weitzman D, Davidovici B, Shapiro J, Cohen AD. Psoriasis and dyslipidaemia: a population-based study. Acta Derm Venereol. 2008;88:561-565.

[18] Dotsenko O, Chackathayil J, Patel JV, Gill PS, Lip GY. Candidate circulating biomarkers for the cardiovascular disease continuum. Curr Pharm Des. 2008;14:2445-2461.

[19] Martin Hübner A, Tenbaum SP. Complete remission of palmoplantar psoriasis through Helicobacter pylori eradication: a case report. Clin Exp Dermatol. 2008;33:339-3340.

[20] Ali M, Whitehead M. Clearance of chronic psoriasis after eradication therapy for Helicobacter pylori infection. J Eur Acad Dermatol Venereol. 2008;22:753-754.

[21] Daudén E, Vázquez-Carrasco MA, Peńas PF, Pajares JM, García-Díez A. Association of Helicobacter pylori infection with psoriasis and lichen planus: prevalence and effect of eradication therapy. Arch Dermatol. 2000;136:1275-1276.

[22] Solanki LS, Srivastava N, Singh S. Superantigens: a brief review with special emphasis on dermatologic diseases. Dermatol Online J. 2008;14:3.

[23] Daudén E, Cabrera MM, Ońate MJ, Pajares JM, García-Díez A. CagA seropositivity in Helicobacter pylori positive patients with psoriasis. J Eur Acad Dermatol Venereol. 2004; 18:116-117.

[24] Noah PW, Handorf CR, Skinner RB Jr, Mandrell TD, Rosenberg EW. Skin basement membrane zone: a depository for circulating microbial antigen evoking psoriasis and autoimmunity. Skinmed. 2006;5:72-79.

[25] Prinz JC. Psoriasis vulgaris-a sterile antibacterial skin reaction mediated by cross-reactive $\mathrm{T}$ cells? An immunological view of the pathophysiology of psoriasis. Clin Exp Dermatol. 2001;26:326-332.

[26] Borén T, Falk P, Roth KA, Larson G, Normark S. Attachment of Helicobacter pylori to human gastric epithelium mediated by blood group antigens. Science. 1993;262:1892-1895.

[27] Ilver D, Arnqvist A, Ogren J et al. Helicobacter pylori adhesin binding fucosylated histo-blood group antigens revealed by retagging. Science. 1998;279:373-377. 
[28] Jones AC, Logan RP, Foynes S, Cockayne A, Wren BW, Penn CW. A flagellar sheath protein of Helicobacter pylori is identical to $\mathrm{HpaA}$, a putative $\mathrm{N}$-acetylneuraminyllactose-binding hemagglutinin, but is not an adhesin for AGS cells. $J$ Bacteriol. 1997; 179:5643-5647.

[29] Valkonen KH, Wadström T, Moran AP. Interaction of lipopolysaccharides of Helicobacter pylori with basement membrane protein laminin. Infect Immun. 1994;62:3640-3648.

[30] Humbert P, Bidet A, Treffel P, Drobacheff C, Agache P. Intestinal permeability in patients with psoriasis. $J$ Dermatol Sci. 1991:2:324-326.

[31] Fukuda Y, Bamba H, Okui M et al. Helicobacter pylori infection increases mucosal permeability of the stomach and intestine. Digestion 2001;63:93-96.

[32] Kurose I, Granger DN, Evans DJ Jr et al. Helicobacter pyloriinduced microvascular protein leakage in rats: role of neutrophils, mast cells, and platelets. Gastroenterology. 1994; 107:70-79.

[33] Zito F, Di Castelnuovo A, D'Orazio A et al. Helicobacter pylori infection and the risk of myocardial infarction: role of fibrinogen and its genetic control. Thromb Haemost. 1999; 82:14-18.

[34] Elizalde JI, Gómez J, Panés J et al. Platelet activation In mice and human Helicobacter pylori infection. J Clin Invest. 1997; 100:996-1005.

[35] Bokemeyer B. Asymptomatic elevation of serum lipase and amylase in conjunction with Crohn's disease and ulcerative colitis. Z Gastroenterol. 2002;40:5-10.

[36] Argiris A, Mathur-Wagh U, Wilets I, Mildvan D. Abnormalities of serum amylase and lipase in HIV-positive patients. $\mathrm{Am}$ J Gastroenterol. 1999;94:1248-1252.

[37] Brauchli YB, Jick SS, Meier CR. Psoriasis and the risk of incident diabetes mellitus: a population-based study. $\mathrm{Br} J$ Dermatol. 2008;159:1331-1337.

[38] Badalov N, Baradarian R, Iswara K, Li J, Steinberg W, Tenner S. Drug-induced acute pancreatitis: an evidence-based review. Clin Gastroenterol Hepatol. 2007;5:648-661.

[39] Toubanakis C, Batziou E, Sipsas N, Galanopoulos G, Tzivras M, Archimandritis A. Acute pancreatitis after long-term therapy with mesalazine, and hyperamylasaemia associated with azathioprine in a patient with ulcerative colitis. Eur J Gastroenterol Hepatol. 2003;15:933-934.

[40] Sato M, Takamura M, Sato Y et al. Drug-induced acute pancreatitis associated with 22-oxacalcitriol ointment for treatment of psoriasis. JOP. 2009;10:336-337.

[41] Igarashi H, Ito T, Yoshinaga M, Oono T, Sakai H, Takayanagi R. Acetaminophen-induced acute pancreatitis. A case report. JOP. 2009;10:550-553.

[42] Famularo G, Minisola G, Nicotra GC, De Simone C. Idiosyncratic pancreatitis associated with perindopril. JOP. 2005;6: 605-607.

[43] Gallego-Rojo FJ, Gonzalez-Calvin JL, Guilarte J, CasadoCaballero FJ, Bellot V. Perindopril-induced acute pancreatitis. Dig Dis Sci. 1997;42:1789-1791.
[44] Nagpal K, Minocha VR, Agrawal V, Kapur S. Evaluation of intestinal mucosal permeability function in patients with acute pancreatitis. Am J Surg. 2006;192:24-28.

[45] Liu H, Li W, Wang X, Li J, Yu W. Early gut mucosal dysfunction in patients with acute pancreatitis. Pancreas. 2008; 36:192-196.

[46] Yuan BS, Zhu RM, Braddock M, Zhang XH, Shi W, Zheng MH. Interleukin-18: a pro-inflammatory cytokine that plays an important role in acute pancreatitis. Expert Opin Ther Targets. 2007;11:1261-1271.

[47] Flisiak I, Klepacki A, Chodynicka B. Plasma and scales levels of interleukin 18 in comparison with other possible clinical and laboratory biomarkers of psoriasis activity. Biomarkers. 2006;11:194-200.

[48] Pietrzak A, Janowski K, Chodorowska G et al. Plasma interleukin-18 and dendritic cells in males with psoriasis vulgaris. Mediators Inflamm. 2007;2007:61254.

[49] Murray ML, Bergstresser PR, Adams-Huet B, Cohen JB. Relationship of psoriasis severity to obesity using same-gender siblings as controls for obesity. Clin Exp Dermatol. 2009;34:140-144.

[50] Kim CH, Younossi ZM. Nonalcoholic fatty liver disease: a manifestation of the metabolic syndrome. Cleve Clin J Med. 2008;75:721-728.

[51] Langman G, Hall PM, Todd G. Role of non-alcoholic steatohepatitis in methotrexate-induced liver injury. J Gastroenterol Hepatol. 2001;16:1395-1401.

[52] Vecchiet J, Falasca K, Cacciatore P et al. Association between plasma interleukin-18 levels and liver injury in chronic hepatitis $\mathrm{C}$ virus infection and non-alcoholic fatty liver disease. Ann Clin Lab Sci. 2005;35:415-422.

[53] Kern PA, Ranganathan S, Li C, Wood L, Ranganathan G. Adipose tissue tumor necrosis factor and interleukin-6 expression in human obesity and insulin resistance. Am J Physiol Endocrinol Metab. 2001;280:E745-51.

[54] Boehncke WH, Boehncke S. Cardiovascular morbidity in psoriasis: epidemiology, pathomechanisms, and clinical consequences. G Ital Dermatol Venereol. 2008;143:307-313.

[55] Henriksen JH, Gř tze JP, Fuglsang S, Christensen E, Bendtsen F, Mřller S. Increased circulating pro-brain natriuretic peptide (proBNP) and brain natriuretic peptide (BNP) in patients with cirrhosis: relation to cardiovascular dysfunction and severity of disease. Gut. 2003;52:1511-1517.

[56] Muscari A, Berzigotti A, Bianchi G et al. Non-cardiac determinants of NT-proBNP levels in the elderly: relevance of haematocrit and hepatic steatosis. Eur J Heart Fail. 2006;8: 468-476.

[57] Bayes-Genis A, DeFilippi C, Januzzi JL Jr. Understanding amino-terminal pro-B-type natriuretic peptide in obesity. Am J Cardiol. 2008;101:89-94.

Submitted:15 April, 2009 Accepted after reviews: 22 July, 2009 